\title{
Use of a Flexible Cryoprobe for Removal of Tracheobronchial Blood Clots
}

\author{
Inderpaul Singh Sehgal MD DM, Sahajal Dhooria MD DM, Ritesh Agarwal MD DM, \\ and Digambar Behera MD
}

\section{Introduction}

Acute obstruction of the central airways can occur due to foreign-body inhalation, mucus plugs, or blood clots. Airway obstruction due to blood clots is clinically suspected in a patient with acute respiratory failure if the patient has hemoptysis. However, one third of patients with acute endobronchial obstruction due to blood clots may present without obvious hemoptysis. ${ }^{1,2}$ Management involves removal of the clot by bronchoscopic (flexible or rigid) extraction. ${ }^{1}$ During flexible bronchoscopy, clots can be removed piecemeal by biopsy forceps, dislodged using a Fogarty catheter, or removed en bloc using either suctioning or a cryoprobe. ${ }^{3}$

A cryoprobe creates very low temperatures using the Joule-Thomson effect, wherein sudden expansion of a gas from a high-pressure region to a low-pressure region results in cooling. This leads to freezing of the object or tissue. Cryotherapy has been used predominantly in the ablation of benign (granulation tissue, carcinoid, airway strictures) and malignant (bronchogenic carcinoma, salivary gland tumors of the trachea) lesions of the airways and for cryorecanalization (restoring the patency of an airway by removing a tumor). Other uses include endobronchial and transbronchial lung biopsies. ${ }^{4}$ Removal of certain foreign bodies such as tablets, food particles, and peanuts by cryoextraction has also been described. ${ }^{5}$ Herein, we report the cryoextraction of a large endobronchial blood clot using a cryoprobe during flexible bronchoscopy.

The authors are affiliated with the Department of Pulmonary Medicine, Postgraduate Institute of Medical Education and Research, Chandigarh, India.

The authors have disclosed no conflicts of interest.

Correspondence: Sahajal Dhooria MD DM, Department of Pulmonary Medicine, Postgraduate Institute of Medical Education and Research, Chandigarh 160012, India. E-mail: sahajal@gmail.com.

DOI: $10.4187 /$ respcare.03861

\section{Case Summary}

A 60-y-old woman was admitted to the emergency room with a diagnosis of stroke. On evaluation, she was found to have atrial fibrillation with a fast ventricular rate. Physical examination revealed right-sided hemiplegia. The Glasgow coma score was $12 / 15$, and she had a poor cough reflex. She was managed with antiplatelet therapy and was given heparin for deep venous thrombosis prophylaxis. During her hospital stay, she was intubated and subsequently underwent a tracheotomy for airway protection. She had 2 episodes of bleeding during tracheal suctioning, following which she developed respiratory failure. She required an oxygen flow of $15 \mathrm{~L} / \mathrm{min}$ through the tracheostomy tube to maintain normal arterial oxygen saturation. Chest radiography revealed a complete left-lung collapse (Fig. 1). An urgent flexible bronchoscopy (BF-1T150, 2.6-mm internal diameter, Olympus, Tokyo, Japan) was performed through the tracheal stoma, revealing a large blood clot along with inspissated mucus that was completely occluding the left main bronchus. Several attempts to dislodge the clot with suctioning and biopsy forceps were unsuccessful, and only small pieces could be retrieved. Due to failure of conventional methods, we used a cryoprobe to dislodge the clot. The cryoprobe $(1.9-\mathrm{mm}$ outer diameter, 780-mm length, ERBE Elektromedizin, Tübingen, Germany) was introduced through the working channel of the bronchoscope. The probe was applied to the clot, and the probe tip was cooled for $15 \mathrm{~s}$. The blood clot, cryoprobe, and bronchoscope were then removed en bloc (Fig. 2). The cryoextraction procedure was performed in $\sim 7 \mathrm{~min}$. Two h after the procedure, the patient's hypoxemia had resolved. A chest radiograph revealed a fully expanded left lung (Fig. 3).

\section{Discussion}

This case highlights the successful use of a cryoprobe in the management of airway obstruction during flexible bronchoscopy. The index case presented with acute respiratory failure resulting from left-lung collapse secondary to a 


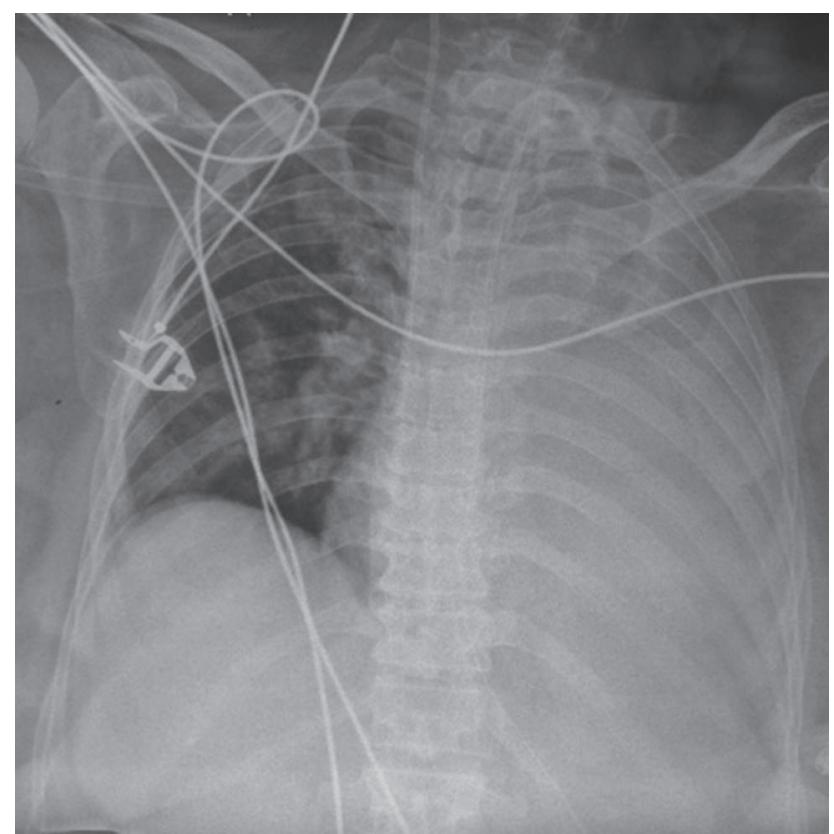

Fig. 1. Chest radiograph of the patient before bronchoscopy depicting left-lung collapse.

blood clot, which was removed by cryoextraction, leading to resolution of respiratory failure.

Airway obstruction from endobronchial blood clots can occur in several disorders. These include both benign (pulmonary tuberculosis, bronchiectasis, vasculitides, invasive fungal infections, bleeding disorders, post-tracheotomy, trauma during suctioning, and others) and malignant (bronchogenic carcinoma, carcinoid tumors, metastatic pulmonary nodules, and others) conditions. Endobronchial blood clots may be preceded by sentinel hemoptysis (mild or massive); however, $\sim 30 \%$ of patients may present with respiratory failure without a prior history of hemoptysis. ${ }^{1,2}$ Treatment involves hemodynamic stabilization, identification of the cause of hemoptysis, control of bleeding, and removal of the endobronchial clot (if causing respiratory failure). In our case, the cause of bleeding was the use of antiplatelet therapy for treatment of the cerebrovascular accident, which complicated a recent tracheotomy performed for airway protection.

Removal of endobronchial clots can be accomplished by either flexible or rigid bronchoscopy. During flexible bronchoscopy, clot retrieval can be performed piecemeal using biopsy forceps or by withdrawal of the bronchoscope with the clot adhering to the tip through continuous suction. The clot can also be displaced using a Fogarty catheter or lysed with thrombolytic agents, followed by suctioning. ${ }^{1-3,6,7}$ Thrombolytic agents could theoretically pose a risk for rebleeding, whereas manipulation with a Fogarty catheter across the clot is a blind procedure that itself can induce minor trauma. ${ }^{8,9}$ Furthermore, these tech-

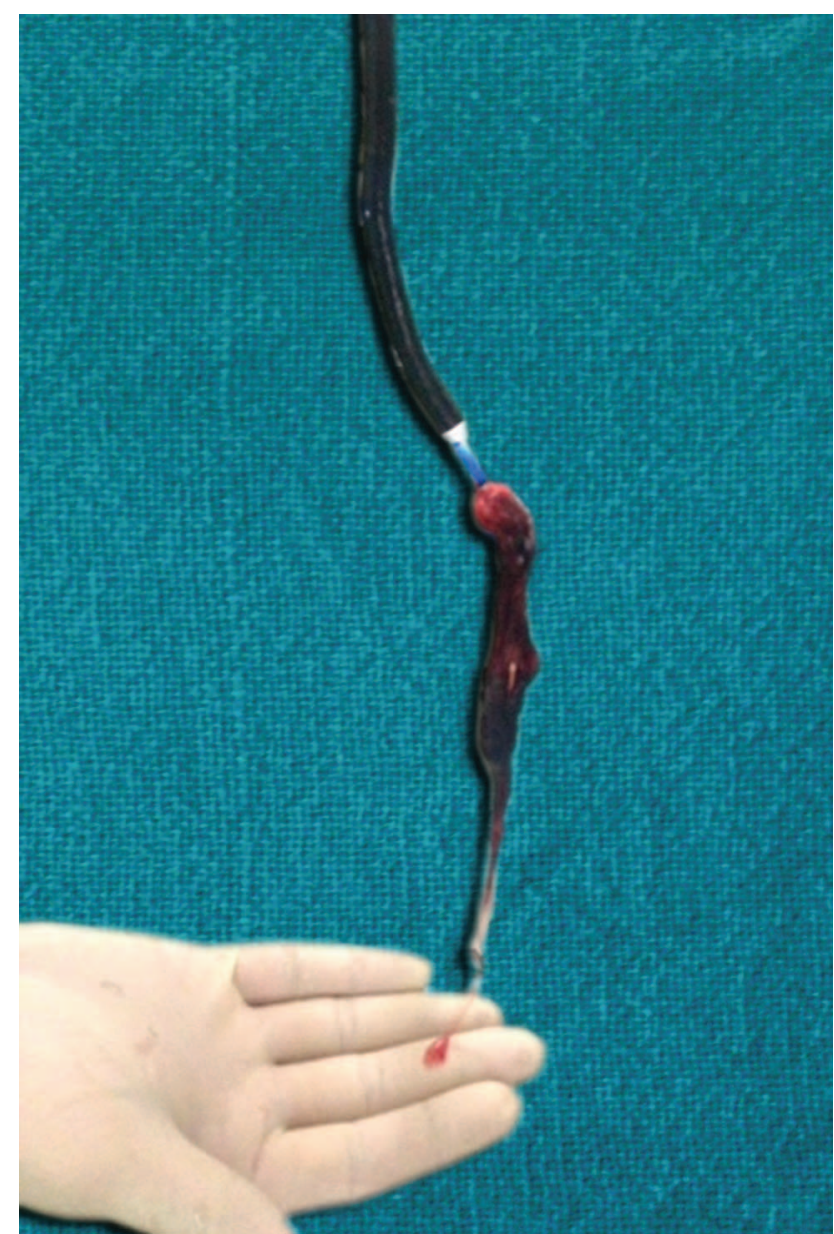

Fig. 2. Flexible bronchoscope and cryoprobe with the blood clot and inspissated mucus attached to the tip of the cryoprobe.

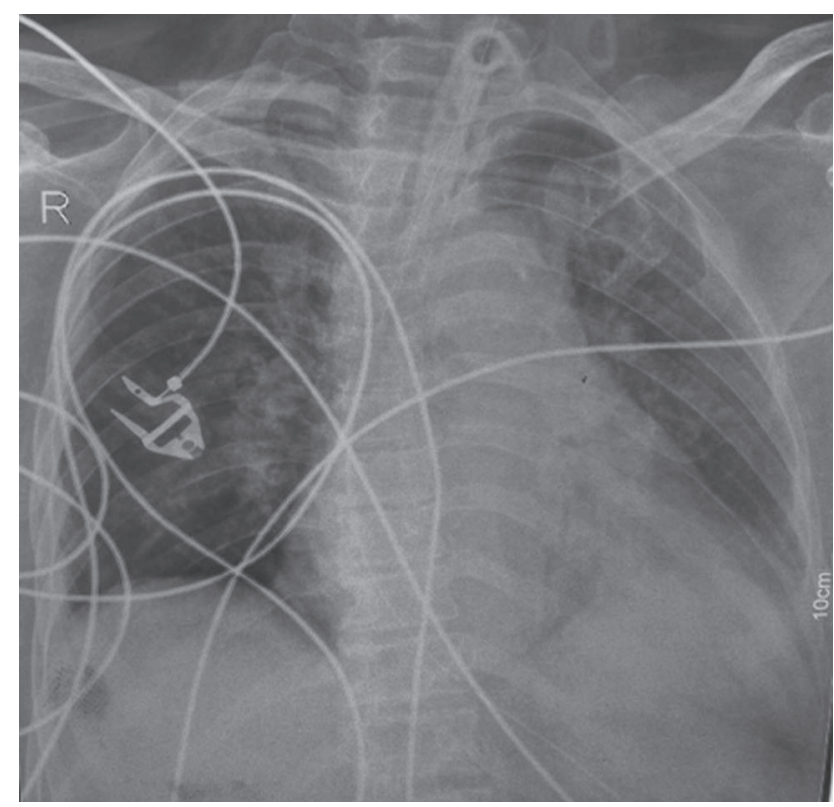

Fig. 3. Chest radiograph after bronchoscopic cryoextraction of the clot showing an expanded left lung. 
Table 1. Case Reports Describing Cryoextraction of Endobronchial Blood Clots

\begin{tabular}{|c|c|c|c|c|c|c|c|}
\hline Reference & Age (y) & Sex & Cause of Bleeding & Location of Clot & $\begin{array}{l}\text { Respiratory } \\
\text { Failure }\end{array}$ & $\begin{array}{c}\text { Type of } \\
\text { Bronchoscope }\end{array}$ & $\begin{array}{c}\text { Duration of } \\
\text { Procedure }\end{array}$ \\
\hline De Weerdt et $\mathrm{al}^{9}$ & 54 & Male & Vascular anomaly & Left main bronchus & Yes & Rigid & NR \\
\hline Gonzalez and Khosla ${ }^{13}$ & 83 & Male & Coagulopathy & Left main bronchus & Yes & Flexible & NR \\
\hline Bhardwaj et al ${ }^{11}$ & 54 & Male & Iatrogenic bronchial mucosal tear & Right main bronchus & Yes & Flexible & NR \\
\hline Lee et $\mathrm{al}^{8}$ & 66 & Female & Thrombocytopenia & Left main bronchus & Yes & Flexible & $15 \mathrm{~min}$ \\
\hline
\end{tabular}

niques take more time; in our case, due to our patient's critical condition with respiratory failure, we needed a procedure that would result in rapid clot extraction. Hence, we chose a cryoprobe, the use of which not only results in immediate improvement but can also cause hemostasis by vasoconstriction and slowing of blood flow. Rigid bronchoscopy has been used in cases in which attempts to retrieve the clot by flexible bronchoscopy failed. However, rigid bronchoscopy involves the use of general anesthesia, which may be associated with an increase in morbidity (and mortality), especially in patients such as this index case. ${ }^{10}$

The use of cryoprobes for successful extraction of endobronchial blood clots has also been described previously (Table 1). ${ }^{3,8,9,11-13}$ There are 2 types of cryoprobes: flexible and rigid. The former can be used through both flexible and rigid bronchoscopes (with a flexible scope inserted through the rigid barrel). Rigid cryoprobes are larger; however, we have found them to have limited utility because they are not truly rigid, and the growth or clots tend to fall off due to lack of stability. Although initially used for management of centrally located airway tumors, the current equipment has widened the scope of cryosurgery in the management of various airway disorders such as removal of foreign bodies, management of benign strictures and granulation tissue, and lung biopsies. ${ }^{4,5,14,15}$ Because cryoprobes can be used for several indications during flexible and rigid bronchoscopy and equipment is not unduly expensive (approximately $\$ 10,000$ ), we believe that cryotherapy units should be part of the diagnostic and therapeutic armamentarium of every interventional pulmonology unit. Furthermore, there is a short learning curve, as it just requires proper maneuvering of the probe, which can be done by any experienced bronchoscopist.

The endobronchial uses of cryotherapy techniques are based on the following principles: (1) cryoablation involving repeated cycles of freezing and thawing, leading to tissue destruction as a result of cryothrombosis and intracellular crystallization, or (2) cryoadherence, wherein the object of interest (tumor, foreign body, or other) adheres to the cryoprobe due to crystallization of the water molecules at the interface. The object can then be extracted en bloc along with the probe, a process called cryoextraction. When it is used for restoring the patency of an airway by removing a tumor, it is called cryorecanalization. The use of a cryoprobe is safe in the airways, as it avoids injury to the relatively cryo-resistant tracheobronchial cartilage. ${ }^{5} \mathrm{Al}-$ though endobronchial objects or foreign bodies with highwater content are ideally suited for cryoextraction, recent evidence suggests that this technique can also be used to extract inorganic objects with low water content. In an ex vivo study involving retrieval of 18 commonly aspirated objects using cryoprobes, many inorganic objects (hairpin, glass, stapler, pill) with a low water content could be removed, indicating the utility of cryoprobes in wider indications than previously thought. ${ }^{16}$ In conclusion, our case demonstrates that bronchoscopic cryoextraction is a simple and safe modality for removal of endobronchial blood clots and can be used to avoid invasive procedures such as rigid bronchoscopy.

\section{Teaching Points}

- Acute respiratory failure can result from endobronchial blood clots, which can be detected by bronchoscopy.

- Tracheobronchial blood clots can be effectively retrieved by cryoextraction during flexible bronchoscopy.

- Flexible bronchoscopy using a cryoprobe can be used in place of rigid bronchoscopy, which requires general anesthesia with its attendant risks.

\section{REFERENCES}

1. Arney KL, Judson MA, Sahn SA. Airway obstruction arising from blood clot: three reports and a review of the literature. Chest 1999; 115(1):293-300.

2. Underwood SM, Summerfield RJ. Obstruction of tracheostomy by thrombus. Crit Care Med 1986;14(9):834.

3. Rafanan AL, Mehta AC. Adult airway foreign body removal. What's new? Clin Chest Med 2001;22(2):319-330.

4. Rubio ER, le SR, Whatley RE, Boyd MB. Cryobiopsy: should this be used in place of endobronchial forceps biopsies? BioMed Res Int 2013;2013:730574.

5. Vergnon JM. How I do it: bronchoscopic cryotherapy. J Bronchology 1995;2(4):323-327.

6. Cetin G, Cörüt R, Küpeli E. Removal of a large endobronchial blood clot using a flexible bronchoscope. J Bronchology Interv Pulmonol 2012;19(4):358-359. 


\section{Cryoextraction of Endobronchial Blood Clot}

7. Vajo Z, Parish JM. Endobronchial thrombolysis with streptokinase for airway obstruction due to blood clots. Mayo Clin Proc 1996; 71(6):595-596.

8. Lee H, Leem CS, Lee JH, Lee CT, Cho YJ. Successful removal of endobronchial blood clots using bronchoscopic cryotherapy at bedside in the intensive care unit. Tuberc Respir Dis 2014;77(4):193196.

9. De Weerdt S, Noppen M, Remels L, Vanherreweghe R, Meysman M, Vincken W. Successful removal of a massive endobronchial blood clot by means of cryotherapy. J Bronchology Interv Pulmonol 2005; 12(1):23-24.

10. Cunanan OS. The flexible fiberoptic bronchoscope in foreign body removal. Experience in 300 cases. Chest 1978;73(5 Suppl):725-726.

11. Bhardwaj B, Bhardwaj H, Youness HA, Awab A. Bronchoscopic cryoextraction: a novel approach for the removal of massive endo- bronchial blood clots causing acute airway obstruction. Southwest J Pulm Crit Care 2013;7:184-189.

12. Homasson JP, Vergnon JM. Cryotherapy to extract obstructing blood clots. J Bronchology Interv Pulmonol 2002;9(2):158-159.

13. Gonzalez EL, Khosla R. Cryotherapy in the management of airway clotting in post coronary artery bypass graft complicated with massive hemoptysis. Am J Respir Crit Care Med 2012;185:A5967.

14. Morgan RK, Ernst A. Cryosurgery. Eur Respir Monogr 2010;48: 161-172.

15. Poletti V, Casoni GL, Gurioli C, Ryu JH, Tomassetti S. Lung cryobiopsies: a paradigm shift in diagnostic bronchoscopy? Respirology 2014;19(5):645-654.

16. Fruchter O, Kramer MR. Retrieval of various aspirated foreign bodies by flexible cryoprobe: in vitro feasibility study. Clin Respir J 2014 [Epub ahead of print] doi: 10.1111/crj.12120. 